\title{
IEDITORIAL
}

\section{Atrial fibrillation in patients with cardiac implantable electronic devices: new perspectives with important clinical implications}

\author{
Giuseppe Boriani, Marco Vitolo \\ Department of Biomedical, Metabolic and Neural Sciences, Division of Cardiology, University of Modena and Reggio Emilia, Policlinico di Modena, Modena, Italy
}

RELATED ARTICLE

by Dębski et al,

see p. 1140

Correspondence to:

Prof. Giuseppe Boriani, PhD,

Cardiology Division, Department of Biomedical, Metabolic and Neural Sciences, University of Modena and Reggio Emilia, Policlinico di Modena, Via del Pozzo 71, 41121 Modena, Italy, phone: +390594225836, email: giuseppe.boriani@unimore.it Received: October 19, 2019. Accepted: October 21, 2019. Published online: December 19, 2019. Kardiol Pol. 2019; 77 (12): 1119-1120 doi:10.33963/KP.15110 Copyright by the Author(s), 2019
Atrial fibrillation (AF) is the most common arrhythmia encountered in clinical practice, and even if it is usually associated with palpitations, it frequently may be asymptomatic (up to $40 \%$ of cases) ${ }^{1}$ or it may present atypical symptoms (around $25 \%$ of cases). ${ }^{2}$ Asymptomatic AF is reported to be more prevalent in men, older patients, and those with permanent AF. Moreover, it is usually associated with more complex comorbidities and an increased risk of thromboembolism as well as cardiovascular and all-cause mortality, as compared with symptomatic AF.1,2

In the current issue of Kardiologia Polska (Kardiol Pol, Polish Heart Journal), Dębski et al ${ }^{3}$ report on an interesting retrospective study that evaluated the incidence of permanent AF in a cohort of 3932 patients implanted with a dual-chamber pacemaker, analyzed the predictors of permanent AF development, and considered the impact of AF on patients' outcome.

The setting of the study is quite interesting since pacemakers with an atrial lead enable a precise and detailed assessment of presence or absence of atrial tachyarrhythmia and allow to quantify, in terms of AF burden, the time spent in $\mathrm{AF}$, as well as the evolution or progression of the arrhythmia, independently of the presence of symptoms or their absence. ${ }^{4,5}$

During a follow-up of 4.6 years, $19 \%$ of patients developed permanent AF and at year 1, 5, 10 , and 15 after device implantation, permanent AF was found in $4.4 \%, 13.3 \%, 25 \%$, and $32.3 \%$ of patients, respectively. This indicates that with time, the burden of AF, either symptomatic or asymptomatic, is substantial, and needs for appropriate decision-making, first of all, to start oral anticoagulation in patients at risk.
Permanent AF was associated with age at baseline, with the risk increasing 2-fold for each decade of life, as well as with male sex. In this retrospective study, the position of RV lead or the type of pacing were not related with permanent AF. The authors highlight the need for follow-up to evaluate the onset of AF that would enable prompt reaction. Unfortunately, the study was not based on continuous cardiac rhythm monitoring since many implanted pacemakers had no automated storage of intracardiac electrocardiograms and most of them were not capable of detecting AF. The extended diagnostic capabilities of modern pacemakers allow continuous monitoring of the cardiac rhythm and appropriate detection of atrial tachyarrhythmias known as atrial high-rate episodes (AHREs). ${ }^{6}$

AHREs, currently defined as episodes of at least 5 minutes of atrial tachyarrhythmias, also including $\mathrm{AF}$, with an atrial rate higher than 175 to $180 \mathrm{bpm}$, are found on follow-up at routine device check or at remote monitoring, and classified in terms of duration of a single episode or time spent in atrial tachyarrhythmias during a day. ${ }^{5,7}$

The extended diagnostic capabilities of implanted devices have led to new terms, such as AF burden, defined as the overall time spent in AF during a specified period of time, and subclinical AF, defined as episodes of atrial tachyarrhythmias with their duration between 5 minutes and 24 hours, detected by a cardiac implantable electronic device in patients without clinical history or symptoms of AF. . $^{89}$

In the ASSERT study (Asymptomatic Atrial Fibrillation and Stroke Evaluation in Pacemaker Patients and the Atrial Fibrillation Reduction 
Atrial Pacing Trial), subclinical atrial tachyarrhythmias that lasted at least 6 minutes were detected with device diagnostics in around 10\% of patients within 3 months after implantation but during a follow-up of 2.5 years; additional subclinical atrial tachyarrhythmias occurred in around $25 \%$ of patients, and around $16 \%$ of those with AHREs developed clinically overt AF. ${ }^{9}$

A literature review by Freedman et al ${ }^{10}$ revealed that AHREs longer than 5 to 6 minutes are common in patients with a cardiac implantable electronic device, with the incidence of $10 \%$ to $68 \%$.

The clinical significance of AHRE is related to the associated risk of stroke. A series of studies, which collected data on more than 22000 patients overall, showed that the burden of AHRE with a duration of 5 to 6 minutes or longer is associated with a 2.4-fold increase in the risk of stroke or systemic thromboembolism (compared with subjects without AF) that is lower than the increased risk commonly reported for clinical AF (4.8-fold). ${ }^{11}$

In view of these findings, the clinical significance of device-detected AHREs is currently debated with regard to the absolute need for prescribing oral anticoagulants, and individualized decision-making is needed before completion of the ongoing prospective trials. ${ }^{10}$

After detection of AHREs, an intensified patient follow-up is recommended, especially if anticoagulation is not instituted, possibly with remote monitoring targeted to detect clinical AF or the transition into AHRE lasting more than 24 hours as well as to detect important clinical changes such as onset or exacerbation of heart failure. ${ }^{6,12,13}$

Aside from the risk of stroke, detection of atrial tachyarrhythmias or overt $\mathrm{AF}$ in patients with pacemakers has important implications for patient outcomes. In a study with a 4-year follow-up, detection of AHREs was associated with an increased risk of thromboembolism and death. ${ }^{14}$

In the study by Dębski et al, ${ }^{3}$ the occurrence of permanent $\mathrm{AF}$ was associated with a significantly increased unadjusted risk of death. In a previous study evaluating a cohort of real-world patients with AF, nonparoxysmal AF had a worse outcome in terms of all-cause mortality, which was related to a more severe patient status. Age, chronic heart failure, chronic kidney disease, and diabetes were independent predictors of an adverse outcome..$^{15}$

In conclusion, nowadays, there are novel clinical perspectives in the field of cardiac pacemakers because they are not only a simple support of the electrical activity in a diseased heart but also constitute advanced diagnostic systems, with remote data transmission that allow extensive monitoring of rhythm abnormalities, and specifically of AF, also with the possibility to monitor parameters related to heart failure. Integration of these records with clinical data could help in appropriate and timely decision-making, and further long-term registries and studies have to be planned in order to provide data on the clinical impact, organizational implications, and patient outcomes associated with these tools.

\section{ARTICLE INFORMATION}

DISCLAIMER The opinions expressed by the author are not necessarily those of the journal editors, Polish Cardiac Society, or publisher.

CONFLICT OF INTEREST GB has received speaker honoraria from Medtronic, Boston, Boehringer, and Bayer, outside of the submitted work. MV declares no conflicts of interest.

OPEN ACCESS This is an Open Access article distributed under the terms of the Creative Commons Attribution-Non6ommercial-NoDerivatives $4.0 \mathrm{In}$ ternational License (CC BY-NC-ND 4.0), allowing third parties to download articles and share them with others, provided the original work is properly cited, not changed in any way, distributed under the same license, and used for noncommercial purposes only. For commercial use, please contact the journal office at kardiologiapolska@ptkardio.pl.

HOW TO CITE Boriani G, Vitolo M. Atrial fibrillation in patients with cardiac implantable electronic devices: new perspectives with important clinical implications. Kardiol Pol. 2019; 77: 1119-1120. doi:10.33963/KP.15110

\section{REFERENCES}

1 Boriani G, Laroche C, Diemberger I, et al. Asymptomatic atrial fibrillation: clinical correlates, management, and outcomes in the EORP-AF Pilot General Registry. Am J Med. 2015; 128: 509-18.e2.

2 Siontis KC, Gersh B], Killian JM, et al. Typical, atypical, and asymptomatic presentations of new-onset atrial fibrillation in the community: characteristics and prognostic implications. Heart Rhythm. 2016; 13: 1418-1424.

3 Dębski M, Ulman M, Ząbek A, et al. Permanent atrial fibrillation in patients with dual-chamber pacemaker. Kardiol Pol. 2019; 77: 1140-1146.

4 Boriani G, Valzania C, Biffi M, et al. Asymptomatic lone atrial fibrillation - how can we detect the arrhythmia? Curr Pharm Des. 2015; 21: 659-666.

5 Boriani G, Glotzer TV, Santini M, et al. Device-detected atrial fibrillation and risk for stroke: an analysis of $>10,000$ patients from the SOS AF project (Stroke prevention Strategies based on Atrial Fibrillation information from implanted devices). Eur Heart J. 2014; 35: 508-516.

6 Freedman B, Camm J, Calkins H, et al; AF-Screen Collaborators. Screening for atrial fibrillation: a report of the AF-SCREEN International Collaboration. Circulation. 2017; 135: 1851-1867.

7 Boriani G, Da Costa A, Quesada A, et al. Effects of remote monitoring on clinical outcomes and use of healthcare resources in heart failure patients with biventricular defibrillators: results of the MORE-CARE multicentre randomized controlled trial. Eur J Heart Fail. 2017; 19: 416-425.

8 Gorenek B, Bax J, Boriani G, et al. Device-detected subclinical atrial tachyarrhythmias: definition, implications and management-an European Heart Rhythm Association (EHRA) consensus document, endorsed by Heart Rhythm Society (HRS), Asia Pacific Heart Rhythm Society (APHRS) and Sociedad Latinoamericana de Estimulación Cardíaca y Electrofisiología (SOLEACE). Europace. 2017; 19: 1556-1578. 9 Healey JS, Connolly SJ, Gold MR, et al. Subclinical atrial fibrillation and the risk of stroke. N Engl J Med. 2012; 366: 120-129.

10 Freedman B, Boriani G, Glotzer TV, et al. Management of atrial high-rate episodes detected by cardiac implanted electronic devices. Nat Rev Cardiol. 2017; 14: 701-714.

11 Mahajan R, Perera T, Elliott AD, et al. Subclinical device-detected atrial fibrillation and stroke risk: a systematic review and meta-analysis. Eur Heart J. 2018; 39: 1407-1415.

12 Lip GYH, Banerjee A, Boriani G, et al. Antithrombotic therapy for atrial fibrillation: CHEST guideline and expert panel report. Chest. 2018; 154: 1121-1201.

13 Boriani G, Glotzer TV, Ziegler PD, et al. Detection of new atrial fibrillation in patients with cardiac implanted electronic devices and factors associated with transition to higher device-detected atrial fibrillation burden. Heart Rhythm. 2018; 15: 376-383.

14 Miyazawa K, Pastori D, Li YG, et al. Atrial high rate episodes in patients with cardiac implantable electronic devices: implications for clinical outcomes. Clin Res Cardiol. 2019; 108: 1034-1041.

15 Boriani G, Laroche C, Diemberger I, et al. 'Real-world' management and outcomes of patients with paroxysmal vs. non-paroxysmal atrial fibrillation in Europe: the EURObservational Research Programme-Atrial Fibrillation (EORP-AF) General Pilot Registry. Europace 2016; 18: 648-657. 